\title{
Can the Transformer Be Used as a Drop-in Replacement for RNNs in Text-Generating GANs?
}

\author{
Kevin Blin \\ Section of Communication Systems \\ Bachelor semester 6 \\ EPFL, Lausanne, Switzerland \\ kevin.blin@epfl.ch
}

\author{
Andrei Kucharavy \\ Distributed Computing Laboratory \\ EPFL, Lausanne, Switzerland \\ andrei . kucharavy@epfl . ch
}

\begin{abstract}
In this paper we address the problem of fine-tuned text generation with a limited computational budget. For that, we use a well-performing text generative adversarial network (GAN) architecture - DiversityPromoting GAN (DPGAN), and attempted a drop-in replacement of the LSTM layer with a self-attention-based Transformer layer in order to leverage their efficiency. The resulting Self-Attention DPGAN (SADPGAN) was evaluated for performance, quality and diversity of generated text and stability. Computational experiments suggested that a transformer architecture is unable to drop-in replace the LSTM layer, under-performing during the pre-training phase and undergoing a complete mode collapse during the GAN tuning phase. Our results suggest that the transformer architecture need to be adapted before it can be used as a replacement for RNNs in text-generating GANs.
\end{abstract}

\section{Introduction}

Since the introduction of the Transformer in late 2017 by Vaswani et al. (2017), pure self-attention architectures have become ubiquitous in the natural language processing community.

Initially introduced by Bahdanau et al. (2015), the self-attention mechanism proved to be particularly useful in the context of neural machine translation to augment existing recurrent neural networks (RNNs). Recurrent neural networks (RNNs), introduced by Rumelhart et al. (1986) and expanded by Hochreiter and Schmidhuber (1997); Cho et al. (2014), were the state of the art in learning and generating sequential models, notably for texts. With the addition of the attention mechanism, in the context of machine translation, they could focus better on the words and word combinations corresponding to the same concepts in different languages and focus on learning equivalences between them as opposed to trying to infer them directly from the whole excerpts of parallel texts used for learning.

Following the initial introduction of the attention mechanism to augment the RNNs, architectures combining the two became de-facto state of the art. Stacking RNN layers, adding pass-through mechanisms and separating the architecture into encoder and decoder with an attention layer in the middle became a standard, powering among others Google's Neural Machine Translation system (Wu et al., 2016; Johnson et al., 2017). Despite impressive performance, those architectures had a fundamental limitation to their ability to scale. The RNN training and evaluation are sequential by their nature, which means that architectures relying on the RNNs could hardly benefit from the arrival of massively parallel computing.

The innovation of the Transformer was to show that it was possible to learn sequence-to-sequence mapping while dispensing entirely with RNN layers, using only self-attention mechanisms ("Attention is all you need") - Vaswani et al. (2017)). By stacking several layers of self-attention networks to form an encoder and a decoder, as well as introducing multi-head architectures, where each layer of self-attention network could be trained in parallel, the Transformer and architectures derived from it scaled up easily and could be trained in parallel at a scale that was previously unreachable.

That scalability enabled a continuous ramp-up of performance through parameter and training dataset size increase (Brown et al., 2020), which eventually hurt itself against the limitations of reasonable demands on computational power in deployments. In turn, this led to an extensive research into making Transformer-based architectures more efficient, focusing at first on specific instances (Jiao et al., 2020; Sanh et al., 2019) and more recently on more general approaches (eg. Li et al. (2020); 
Mandava et al. (2020); Fedus et al. (2021); Ren et al. (2021)). Combined with the existence of specialized, energy-efficient hardware that is highly compatible with the Transformer architectures, this make Transformer-based architectures an attractive architecture to get the best value of limited computational budget.

The text-generating capabilities of the Transformer also gave rise to a new generation of models specialized in text generation. Rather than mapping texts between languages, they focused on mapping a prompt to a text that would follow it. Google's BERT model (Devlin et al., 2019) isolated and scaled up the Transformer encoder stack in order to perform masked training - predicting masked words in a text - referred to as autoencoding models (Rumelhart et al., 1986; Hinton et al., 2006; Erhan et al., 2010). On the other side of the spectrum, OpenAI's generative pre-training (GPT) family of models (Radford et al., 2018, 2019; Brown et al., 2020), focused on approximating the decoder stack and using the tokens from the prompt in order to initialize the hidden state of the self-attention modules stack - referred to as autoregressive models (by similarity with statistical autoregressive methods (Yule, 1926; Wold, 1938; Slutzky, 1937; Box and Jenkins, 1970), see Bowman et al. (2016) for machine learning applications).

Despite their impressive performance, when it comes to the text generation, both types of models are essentially autoregressive and are trained by max likelihood methods with regards to the training datasets. This poses several challenges. First, the autoregressive models learn the next token as a continuation of real texts they encounter in training, yet during the generation phase they continue from the text they themselves have generated. This means that during the generation phase they can rapidly go off the deeper end into an uncharted territory, they don't have a statistical model, and start generating degenerate output - a problem referred to as exposure biais (Holtzman et al., 2020). So far, solutions to this problem, such as scheduled sampling (Bengio et al., 2015), are far from perfect and result in less diverse sampling and mode collapse (Huszar, 2015). On top of that, the autoregressive nature of the model means that even in the territory where it has learned an appropriate token distribution, it will still be learning potentially undesirable biases (Hutchinson et al., 2020), with no means to correct them other than to curate the entire dataset used to train the model (reviewed in depth by Bender et al. (2021)).

Trying to learn the explicit statistical structure of natural language is, however, not the only way to train generative models. Adversarial Generative Networks (GANs) are a different training mode, where a generative model learns to generate outputs that are indistinguishable from the ones in the training dataset through a competition with a critic model, trained in tandem with it. Introduced by (Goodfellow et al., 2014), they are more robust to output degeneration, given that they always train in the generative mode, and require less computational resources than traditional auto regressive models. Besides, a number of different pre-trained critics can be used to eliminate undesired biases or on the contrary, introduce desired ones, such as specifying an artistic style of an image (Gatys et al., 2015).

The adversarial learning approach has been highly successful for training image generation models, allowing high-quality image generation (Brock et al., 2019), day-to-night or summerto-winter image translation (Isola et al., 2017), or sketch-to-image translation (Lu et al., 2018). GANs application to text generation, however, remained relatively limited. A major reason for that is that the sampling step leading to discrete and sequential token organization, needed for text generation, is problematic for gradient estimation, which is essential for training GANs. As such, most existing text-generating GANs rely on a max-likelihood autoregressive pre-training with the actual adversarial training phase being short and using a small learning rate, similar to a fine-tuning. Unfortunately such an approach failed to address the shortcomings of the purely autoregressive models they acquire during the pre-training phase.

However, recently de Masson d'Autume et al. (2019) was able to demonstrate that it was possible to train a text-generating GAN architecture from scratch, thus avoiding entirely the problems encountered by autoregressive max-likelihood methods. A curious property of the Scratch-GAN he developed, is that while it seems to solve problems that plagues both RNNs and pure self-attention autoregressive models learning the explicit text token distribution, the authors still opted for RNN blocks, foregoing the advantages of Transformer architectures in NLP and self-attention in image-generating GANs (Zhang et al., 2019). 
Given the massive advantages of the Transformer presents when it comes to training as well as with regards to the amount of research performed to make them more efficient, as well as better capabilities of Transformers compared to GANs, we wanted to know if it was possible to perform a drop-in replacement of RNNs with Transformers. Such a Transformer-based GAN could provide two main benefits: the ability of produce higher quality samples at a reduced computational cost compared to traditional RNN-based GANs, and a more scalable language GAN.

In order to approach this question, we chose to perform an experimental evaluation, based on a classical text-generating GAN - Diversity Promoting GAN (DPGAN), developed by Xu et al. (2018). We chose DPGAN due to its straight-forwards architecture and training mode, similarity of its rewards structure to the state of the art text-generating GANs de Masson d'Autume et al. (2019) and the presence of the maximum likelihood pre-training, that we were expecting to be particularly favorable to the Transformer layers. We refer to the DPGAN with RNN layers replaced by a transformer as Selfattention DPGAN (SADPGAN).

\section{Related Work}

The power of the Transformer architecture in the language modelling tasks and its potential to further improve existing GAN architectures did not escape the attention of the machine learning community. So far, self-attention architectures in GANs focused on image generation tasks. Perhaps the two best known examples are TransGAN (Jiang et al., 2021) and Self-Attention GAN (SAGAN) (Zhang et al., 2019). More recent advances, such as the introduction of Generative Adversarial Transformers by Hudson and Zitnick (2021) have build on the Transformer architecture even further, enabling long-range correlation to improve over the existing state of art, showing a potential for it in the GAN setting. However, a common point between all of them is that they focus on the generation of images, rather than texts, and use only a single encoding layer for self attention or attempt to modify self-attention mechanism to better suite the image generation, diverging from the Transformer architecture.

The approach to text generation by combining the Transformer and GANs that comes closest to ours is the SALSA-Text, developed by Gagnon-
Marchand et al. (2019). SALSA-Text is a textgenerating GAN build around a Transformer, discarding the original layer normalization and replacing it with the spectral layer normalization. In addition to that, SALSA-Text uses a modified Transformer architecture, with less layers and a different structure, as well as a specific training regimen, meaning it is more of a GAN built around a Transformer rather than a GAN where an RNN layer has been replaced with a pure self-attention based layer.

Here, we examine the Transformer applicability as a general-purpose element that can be drop-in in architectures requiring a latent space encoding and thus directly replace RNNs structures as LSTMs and Gated Recurrent Units (GRUs).

\section{Contribution and Outline}

Our contribution consisted in assessing the potential of Transformers as a drop-in replacement of LSTMs in text-generating GANs. Through three different experiment involving SADPGAN, we showed that Transformers, despite achieving remarkable results in several NLP tasks, fail to adapt to the adversarial learning and adversarial fine-tuning context (Jeddi et al., 2020), causing SADPGAN to consistently come short of the DPGAN performance and to exhibit severe mode collapse. This results suggest that as of now, the Transformer can't be directly used as a replacement for LSTM without further architectural and training mode changes.

\section{Methodology}

Our work is built upon an existing PyTorch implementation of DPGAN by Liu et al. (2020b).

We used an iterative approach, first implementing a layer using exclusively Transformer encoders, then adding the decoder stack with masking and finally adding teacher forcing during training. For each of these steps we compared the training results to the original implementation.

Since our goal was to investigate the possibility of replacing LSTM layers in text-generating GANs by dropping in Transformer layers, and not to achieve new state of the art for text-generating GANs, we kept our models relatively small: embedding and hidden dimension of 32, Transformer encoder/decoder with two layers, each with 4 attention heads of size 64 .

The training loop for both GANs consisted in 
120 iterations of MLE pre-training for the generator followed by another 120 epochs of adversarial training between the discriminator and generator. To assess the performance of both architectures, we used two negative log likelihood metrics, $N L L_{g e n}$ and $N L L_{d i v}$ which measure the quality and respectively diversity of the generated text and (self)BLEU scores when using real data. For the NLL metrics lower values correspond to better results while for BLEU higher scores are desirable. The code developed for this project is available from https://github. com/TheBlueHawk/RANLP21-70, specifically the RANLP-2021 release. For the ease of use, it was packaged integrated into the code from Liu et al. (2020b).

\section{Results and Discussion}

\subsection{Experiment \#1}

Following the example set by Jiang et al. (2021) in image GANs, we first tried to drop-in a Transformer encoder block, ensuring that it would properly fit the rest of the GAN architecture. For this preliminary experiment we trained both SADPGAN and DPGAN on a synthetic dataset with a vocabulary of 5000 words.

As shown in figure 1, for SADPGAN we didn't observe any improvement during MLE training. This can be explained by the choice of architecture: using only Transformer encoders without combining them with up-sampling layers for image generation or using bidirectional attention heads as in Devlin et al. (2019), doesn't allow the model to correctly learn how to produce realistic samples.

\subsection{Experiment \#2}

Following the results of the first experiment, we modified the Transformer block by adding a Transformer decoder after the Transformer encoder. Contrary to an encoder-only architecture, the addition of the decoder require a second input, the output vector (also know as target), which is fed to the first masked multi-head attention layer of the decoder. To leave a maximum freedom to the decoder when generating sentences, we decided to feed it with an empty vector. For this second experiment we trained both model on real data, specifically the Lin et al. (2014) annotation dataset using a pretrained Word2Vec embedding. Word samples were obtained in parallel using multinomial sampling.

The set of modifications permitted SADPGAN
Figure 1: $N L L_{d i v}$ and $N L L_{g e n}$ losses of SADPGAN and DPGAN during the first experiment.
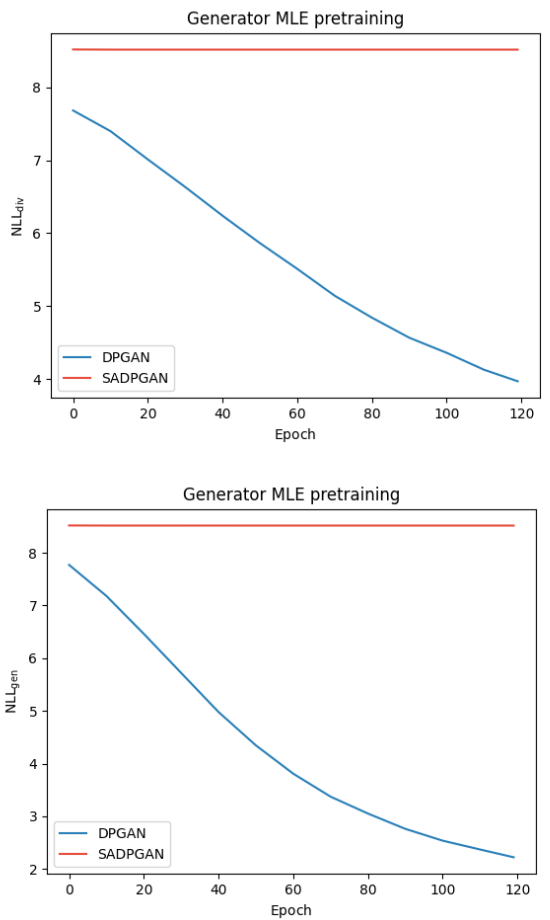

to improve the quality of the generated text during the MLE pre-training iterations but the learning curve flattened out rather quickly and performed worse than the original implementation, with also a decrease of output diversity. During the adversarial training we observed a severe mode collapse: the original DPGAN produced very similar sentences while SADPGAN eventually produced exclusively empty sentences. See table 1 for some samples randomly drawn at the end of pre-training and adversarial training.

The reason for this particular behaviour lies in the role of the target vector, which act as a ground truth in a teacher forcing scenario. This means that parallel sampling cannot be performed without forcing the use of the empty sentence as a reference.

\subsection{Experiment \#3}

To address the problems of experiment \#2 we used two modifications: a new target vector and autoregressive sampling. As a target vector we used the input sentence to the encoder but shifted right by one position: this allowed a correct implementation of teacher forcing during the training phase. During the evaluation phase or when sampling, we don't want the teacher to influence the output of 


\begin{aligned} & \hline DPGAN \\ & pre a person sits on black motorcycle on a busy bench near the side \\ & adv a man riding a motorcycle down a street \\ & SADPGAN \\ & pre flies a various a trash a while begs area in refrigerator \\ & adv "empty sentence" \\ & \hline\end{aligned}

Table 1: Text samples produced after pre-training (pre) and adversarial training (adv) by GAN architectures

Figure 2: $N L L_{d i v}$ and $N L L_{\text {gen }}$ losses of SADPGAN and DPGAN during the second experiment.
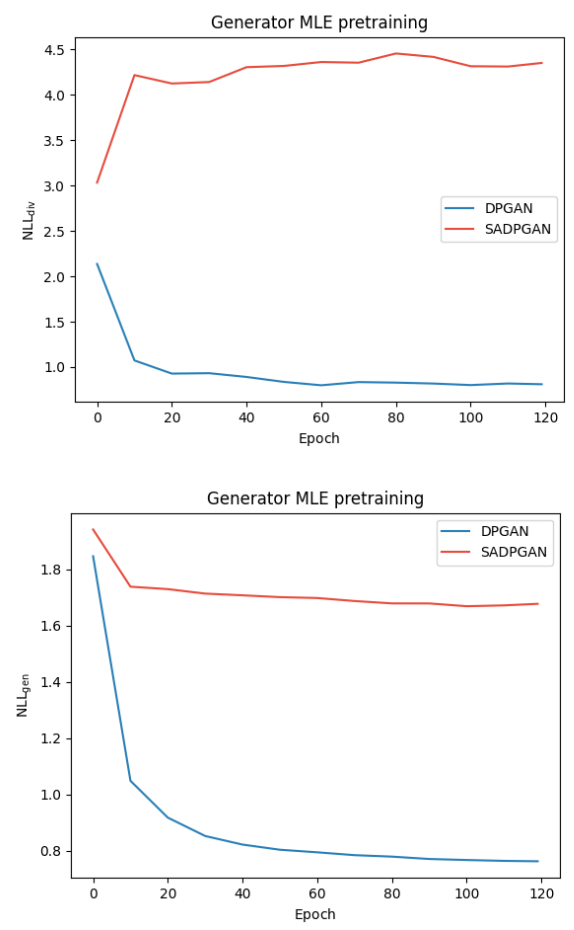

the block, and thus we used a word-by-word autoregressive structure where the previously generated text is reused as the input of the target vector of the Transformer decoder.

The results of pre-training show a greater improvement of the quality of samples but at the cost of severe lack of diversity, resulting in common words repeated in sequence. Again, the adversarial training further exacerbate the issues resulting in total mode collapse. BLEU and self-BLEU scores for different $n$-grams at the end of pre-training and adversarial training are reported in table 2 for both DPGAN and SADPGAN.

\section{Conclusion}

Following our results, we have observed that a Transformer architecture cannot be used as a simple drop-in replacement for RNNs in the context of
Figure 3: $N L L_{d i v}$ and $N L L_{g e n}$ losses of SADPGAN and DPGAN during the third experiment
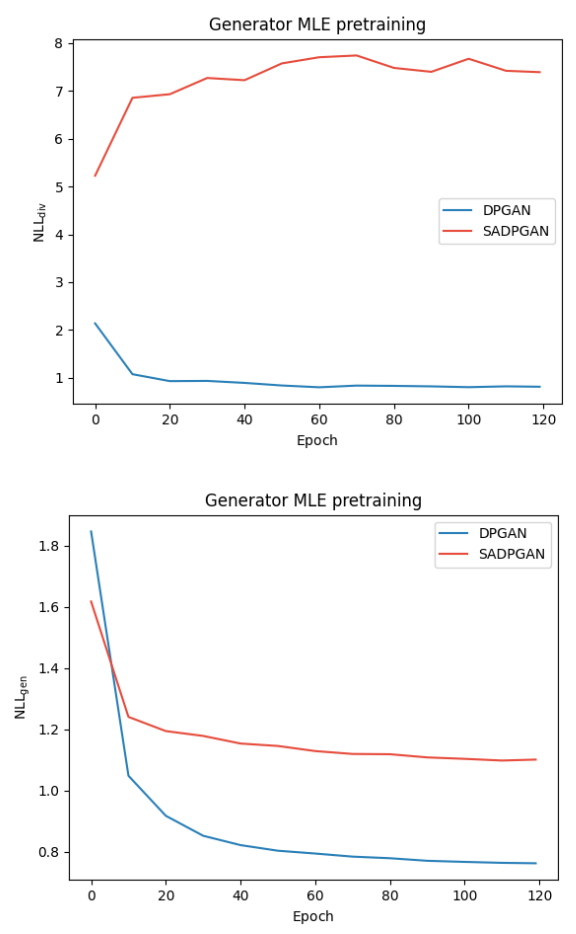

text-generating GANs, at least not in its unmodified form.

This result is not entirely surprising. Despite a great performance of the Transformer and its derivatives in different NLP tasks, Transformerbased architectures are all but simple to train, as reviewed in Liu et al. (2020a).

Another problem with Transformer architectures is their tendency to overfit the distribution of the text they are learning and fail to generate novel text in case insufficiently diverse and varied training datasets are used. In the case of the DPGAN, the small size of the training dataset and the relatively small size of batches likely place us directly into the overfitting territory. Upon the transition into the adversarial training, this overfitting is likely mutually amplified, leading to more and more degenerate text outputs. 


\begin{tabular}{|c|c|c|c|c|c|c|c|}
\hline & \multicolumn{4}{|c|}{ BLEU } & \multicolumn{3}{|c|}{ Self-BLEU } \\
\hline & $n=2$ & $n=3$ & $n=4$ & $n=5$ & $n=2$ & $n=3$ & $n=4$ \\
\hline \multicolumn{8}{|l|}{ DPGAN } \\
\hline pre & 0.73 & 0.497 & 0.306 & 0.185 & 0.77 & 0.537 & 0.327 \\
\hline $\operatorname{adv}$ & 0.845 & 0.737 & 0.578 & 0.455 & 0.993 & 0.989 & 0.982 \\
\hline \multicolumn{8}{|l|}{ SADPGAN } \\
\hline pre & 0.276 & 0.065 & 0.03 & 0.019 & 0.97 & 0.945 & 0.912 \\
\hline $\mathrm{adv}$ & \multicolumn{4}{|c|}{ nan } & \multicolumn{3}{|c|}{ nan } \\
\hline
\end{tabular}

Table 2: n-gram BLEU and self-BLEU scores for DPGAN and SADPGAN after pre-training and adversarial training

A more fundamental problem is that in DPGAN, we are still faced with the transition from the maxlikelihood training regime to a generative regime. Which means that even with improved datasets, the generator is likely to wander off into the uncharted territory as it tries to generate a new token based on the tokens it has already generated rather than tokes sampled from the training dataset. While this problem is present as well with the RNNs, it seems that the output quality decay occurs faster with the Transformer-based architecture.

Despite the existing wealth of text-generating GANs, none, except for SALSA-text (GagnonMarchand et al., 2019) are Transformer-based, but persistently use RNNs, notably LSTM layers, including in the most recent, state-of-the-art ones, such as (de Masson d'Autume et al., 2019). In case of SALSA-text, a modified architecture and a different regularization methods are used, both in two specific setups. It seems that part of this trend in using RNNs rather than a Transformer-based architecture is rooted in the existence of fundamental differences in the way the Transformer learns compared to the RNNs, that make it particularly vulnerable to the hazards of adversarial training regiments.

As a result, we expect that developing text GAN architectures using self-attention based architectures instead of RNNs ones would require designing new GAN architecture from scratch to ensure a initialization and evaluation/reward structure that would be compatible with the the Transformer layer. A pure self-attention layer capable to be a dropin replacement for RNNs is still to be developed. Given the similarity between the adversarial phase occurring in the majority of current text-generating GANs and the adversarial fine-tuning mechanism (reviewed by eg. Jeddi et al. (2020)), we expect that text-generating model fine-tuning to avoid undesirable patterns and adversarial prompts (such as exemplified in Bender et al. (2021)) would not be straightforwards and rely on the development of more robust and stable self-attention architectures.

\section{Future Work}

While this paper presents a negative result, we did not evaluate a number of approaches that could stabilize the training of pure self-attention architectures. Such approaches could prove to be key to the development of self-attention architectures that can be used as drop-in RNN layers replacements.

An interesting avenue is to build upon the achievements of Gagnon-Marchand et al. (2019), and start with a reduced Transformer architecture, combined with a spectral normalization. Building up on this idea, Noise Stability Regularization proposed by Hua et al. (2021), suggested that noise regularization methods were capable to significantly improve the stability pre-trained pure selfattention generative networks fine-tuning, which could indicate an overall improvement in stability that would be visible in generative adversarial training. The regularization approach has been as well highlighted by several other publications, such as Nguyen and Salazar (2019) and would be the first line of research to be investigated.

Another angle of attack would be to increase the amount of tokens used for initialization of the Transformer on the generator side as well as to perform the initialization on multiple levels. This approach have been shown to perform well in Shin et al. (2020). Similarly, existing Transformer architectures are known to require learning rate schedulers for training, something that seems to be entirely absent from almost all existing textgenerating GAN architectures. It is possible that 
a learning rate scheduler needs to be incorporated into a Transformer layer for it to be able to become generally applicable. The importance of the learning rate scheduler for Transformers has been extensively documented in the past, notably by Popel and Bojar (2018) and could also be key in stabilizing the training of Transformer-based architectures.

Overall, in the absence of proof that pure selfattention architectures are inherently unstable in the adversarial training context, there is a number of potential approaches to make them work in the context of GANs and leverage their advantages that are to be explored.

\section{Acknowledgments}

We would like to thank the Armasuisse - Swiss Cyber Defence Campus for the Distinguished Post Doctoral Fellowship supporting AK, and for providing the computational infrastructure for experiments, as well as Drs Rachid Guerraoui (EPFL) and Ljiljana Dolamic (CYD/Armasuisse), for oversight and general advice on this project, and finally Fabien Salvi (EPFL) for the technical support regarding the computational infrastructure organization.

\section{References}

Dzmitry Bahdanau, Kyunghyun Cho, and Yoshua Bengio. 2015. Neural machine translation by jointly learning to align and translate. In 3rd International Conference on Learning Representations, ICLR 2015, San Diego, CA, USA, May 7-9, 2015, Conference Track Proceedings.

Emily M. Bender, Timnit Gebru, Angelina McMillanMajor, and Shmargaret Shmitchell. 2021. On the dangers of stochastic parrots: Can language models be too big? In FAccT '21: 2021 ACM Conference on Fairness, Accountability, and Transparency, Virtual Event / Toronto, Canada, March 3-10, 2021, pages 610-623. ACM.

Samy Bengio, Oriol Vinyals, Navdeep Jaitly, and Noam Shazeer. 2015. Scheduled sampling for sequence prediction with recurrent neural networks. In Advances in Neural Information Processing Systems 28: Annual Conference on Neural Information Processing Systems 2015, December 7-12, 2015, Montreal, Quebec, Canada, pages 1171-1179.

Samuel R. Bowman, Luke Vilnis, Oriol Vinyals, Andrew M. Dai, Rafal Józefowicz, and Samy Bengio. 2016. Generating sentences from a continuous space. In Proceedings of the 20th SIGNLL Conference on Computational Natural Language Learning, CoNLL 2016, Berlin, Germany, August 11-12, 2016, pages $10-21$. ACL.
George EP Box and Gwilym M Jenkins. 1970. Time series analysis: forecasting and control. Holden-Day.

Andrew Brock, Jeff Donahue, and Karen Simonyan. 2019. Large scale GAN training for high fidelity natural image synthesis. In 7th International Conference on Learning Representations, ICLR 2019, New Orleans, LA, USA, May 6-9, 2019. OpenReview.net.

Tom B. Brown, Benjamin Mann, Nick Ryder, Melanie Subbiah, Jared Kaplan, Prafulla Dhariwal, Arvind Neelakantan, Pranav Shyam, Girish Sastry, Amanda Askell, Sandhini Agarwal, Ariel Herbert-Voss, Gretchen Krueger, Tom Henighan, Rewon Child, Aditya Ramesh, Daniel M. Ziegler, Jeffrey Wu, Clemens Winter, Christopher Hesse, Mark Chen, Eric Sigler, Mateusz Litwin, Scott Gray, Benjamin Chess, Jack Clark, Christopher Berner, Sam McCandlish, Alec Radford, Ilya Sutskever, and Dario Amodei. 2020. Language models are few-shot learners. In Advances in Neural Information Processing Systems 33: Annual Conference on Neural Information Processing Systems 2020, NeurIPS 2020, December 6-12, 2020, virtual.

Kyunghyun Cho, Bart van Merrienboer, Çaglar Gülçehre, Dzmitry Bahdanau, Fethi Bougares, Holger Schwenk, and Yoshua Bengio. 2014. Learning phrase representations using RNN encoder-decoder for statistical machine translation. In Proceedings of the 2014 Conference on Empirical Methods in Natural Language Processing, EMNLP 2014, October 25-29, 2014, Doha, Qatar, A meeting of SIGDAT, a Special Interest Group of the ACL, pages 1724-1734. ACL.

Jacob Devlin, Ming-Wei Chang, Kenton Lee, and Kristina Toutanova. 2019. BERT: pre-training of deep bidirectional transformers for language understanding. In Proceedings of the 2019 Conference of the North American Chapter of the Association for Computational Linguistics: Human Language Technologies, NAACL-HLT 2019, Minneapolis, MN, USA, June 2-7, 2019, Volume 1 (Long and Short Papers), pages 4171-4186. Association for Computational Linguistics.

Dumitru Erhan, Yoshua Bengio, Aaron C. Courville, Pierre-Antoine Manzagol, Pascal Vincent, and Samy Bengio. 2010. Why does unsupervised pre-training help deep learning? J. Mach. Learn. Res., 11:625660.

William Fedus, Barret Zoph, and Noam Shazeer. 2021. Switch transformers: Scaling to trillion parameter models with simple and efficient sparsity. CoRR, abs/2101.03961.

Jules Gagnon-Marchand, Hamed Sadeghi, Md. Akmal Haidar, and Mehdi Rezagholizadeh. 2019. SALSATEXT: self attentive latent space based adversarial text generation. In Advances in Artificial Intelligence - 32nd Canadian Conference on Artificial Intelligence, Canadian AI 2019, Kingston, ON, Canada, May 28-31, 2019, Proceedings, volume 
11489 of Lecture Notes in Computer Science, pages 119-131. Springer.

Leon A. Gatys, Alexander S. Ecker, and Matthias Bethge. 2015. A neural algorithm of artistic style. CoRR, abs/1508.06576.

Ian J. Goodfellow, Jean Pouget-Abadie, Mehdi Mirza, Bing $\mathrm{Xu}$, David Warde-Farley, Sherjil Ozair, Aaron C. Courville, and Yoshua Bengio. 2014. Generative adversarial nets. In Advances in Neural Information Processing Systems 27: Annual Conference on Neural Information Processing Systems 2014, December 8-13 2014, Montreal, Quebec, Canada, pages 2672-2680.

Geoffrey E. Hinton, Simon Osindero, and Yee Whye Teh. 2006. A fast learning algorithm for deep belief nets. Neural Comput., 18(7):1527-1554.

Sepp Hochreiter and Jürgen Schmidhuber. 1997. Long short-term memory. Neural Comput., 9(8):17351780 .

Ari Holtzman, Jan Buys, Li Du, Maxwell Forbes, and Yejin Choi. 2020. The curious case of neural text degeneration. In 8th International Conference on Learning Representations, ICLR 2020, Addis Ababa, Ethiopia, April 26-30, 2020. OpenReview.net.

Hang Hua, Xingjian Li, Dejing Dou, Cheng-Zhong Xu, and Jiebo Luo. 2021. Noise stability regularization for improving BERT fine-tuning. In Proceedings of the 2021 Conference of the North American Chapter of the Association for Computational Linguistics: Human Language Technologies, NAACL-HLT 2021, Online, June 6-11, 2021, pages 3229-3241. Association for Computational Linguistics.

Drew A. Hudson and C. Lawrence Zitnick. 2021. Generative adversarial transformers. CoRR, abs/2103.01209.

Ferenc Huszar. 2015. How (not) to train your generative model: Scheduled sampling, likelihood, adversary? CoRR, abs/1511.05101.

Ben Hutchinson, Vinodkumar Prabhakaran, Emily Denton, Kellie Webster, Yu Zhong, and Stephen Denuyl. 2020. Social biases in NLP models as barriers for persons with disabilities. In Proceedings of the 58th Annual Meeting of the Association for Computational Linguistics, ACL 2020, Online, July 5-10, 2020, pages 5491-5501. Association for Computational Linguistics.

Phillip Isola, Jun-Yan Zhu, Tinghui Zhou, and Alexei A. Efros. 2017. Image-to-image translation with conditional adversarial networks. In 2017 IEEE Conference on Computer Vision and Pattern Recognition, CVPR 2017, Honolulu, HI, USA, July 21-26, 2017, pages 5967-5976. IEEE Computer Society.
Ahmadreza Jeddi, Mohammad Javad Shafiee, and Alexander Wong. 2020. A simple fine-tuning is all you need: Towards robust deep learning via adversarial fine-tuning. CoRR, abs/2012.13628.

Yifan Jiang, Shiyu Chang, and Zhangyang Wang. 2021. Transgan: Two transformers can make one strong GAN. CoRR, abs/2102.07074.

Xiaoqi Jiao, Yichun Yin, Lifeng Shang, Xin Jiang, Xiao Chen, Linlin Li, Fang Wang, and Qun Liu. 2020. Tinybert: Distilling BERT for natural language understanding. In Proceedings of the 2020 Conference on Empirical Methods in Natural Language Processing: Findings, EMNLP 2020, Online Event, 16-20 November 2020, volume EMNLP 2020 of Findings of ACL, pages 4163-4174. Association for Computational Linguistics.

Melvin Johnson, Mike Schuster, Quoc V. Le, Maxim Krikun, Yonghui Wu, Zhifeng Chen, Nikhil Thorat, Fernanda B. Viégas, Martin Wattenberg, Greg Corrado, Macduff Hughes, and Jeffrey Dean. 2017. Google's multilingual neural machine translation system: Enabling zero-shot translation. Trans. Assoc. Comput. Linguistics, 5:339-351.

Zhuohan Li, Eric Wallace, Sheng Shen, Kevin Lin, Kurt Keutzer, Dan Klein, and Joseph E. Gonzalez. 2020. Train large, then compress: Rethinking model size for efficient training and inference of transformers. CoRR, abs/2002.11794.

Tsung-Yi Lin, Michael Maire, Serge J. Belongie, Lubomir D. Bourdev, Ross B. Girshick, James Hays, Pietro Perona, Deva Ramanan, Piotr Dollár, and C. Lawrence Zitnick. 2014. Microsoft COCO: common objects in context. CoRR, abs/1405.0312.

Liyuan Liu, Xiaodong Liu, Jianfeng Gao, Weizhu Chen, and Jiawei Han. 2020a. Understanding the difficulty of training transformers. In Proceedings of the 2020 Conference on Empirical Methods in Natural Language Processing, EMNLP 2020, Online, November 16-20, 2020, pages 5747-5763. Association for Computational Linguistics.

Zhiyue Liu, Jiahai Wang, and Zhiwei Liang. 2020b. Catgan: Category-aware generative adversarial networks with hierarchical evolutionary learning for category text generation. In Proceedings of the AAAI Conference on Artificial Intelligence.

Yongyi Lu, Shangzhe Wu, Yu-Wing Tai, and ChiKeung Tang. 2018. Image generation from sketch constraint using contextual GAN. In Computer $\mathrm{Vi}$ sion - ECCV 2018 - 15th European Conference, Munich, Germany, September 8-14, 2018, Proceedings, Part XVI, volume 11220 of Lecture Notes in Computer Science, pages 213-228. Springer.

Swetha Mandava, Szymon Migacz, and Alex Fit Florea. 2020. Pay attention when required. CoRR, abs/2009.04534. 
Cyprien de Masson d'Autume, Shakir Mohamed, Mihaela Rosca, and Jack W. Rae. 2019. Training language gans from scratch. In Advances in Neural Information Processing Systems 32: Annual Conference on Neural Information Processing Systems 2019, NeurIPS 2019, December 8-14, 2019, Vancouver, BC, Canada, pages 4302-4313.

Toan Q. Nguyen and Julian Salazar. 2019. Transformers without tears: Improving the normalization of self-attention. CoRR, abs/1910.05895.

Martin Popel and Ondrej Bojar. 2018. Training tips for the transformer model. Prague Bull. Math. Linguistics, 110:43-70.

Alec Radford, Karthik Narasimhan, Tim Salimans, and Ilya Sutskever. 2018. Improving language understanding by generative pre-training.

Alec Radford, Jeffrey Wu, Rewon Child, David Luan, Dario Amodei, and Ilya Sutskever. 2019. Language models are unsupervised multitask learners. OpenAI blog, 1(8):9.

Jie Ren, Samyam Rajbhandari, Reza Yazdani Aminabadi, Olatunji Ruwase, Shuangyan Yang, Minjia Zhang, Dong Li, and Yuxiong He. 2021. Zerooffload: Democratizing billion-scale model training. In 2021 USENIX Annual Technical Conference, USENIX ATC 2021, July 14-16, 2021, pages 551564. USENIX Association.

D. E. Rumelhart, G. E. Hinton, and R. J. Williams. 1986. Learning Internal Representations by Error Propagation, page 318-362. MIT Press, Cambridge, MA, USA.

Victor Sanh, Lysandre Debut, Julien Chaumond, and Thomas Wolf. 2019. Distilbert, a distilled version of BERT: smaller, faster, cheaper and lighter. CoRR, abs/1910.01108.

Taylor Shin, Yasaman Razeghi, Robert L. Logan IV, Eric Wallace, and Sameer Singh. 2020. Autoprompt: Eliciting knowledge from language models with automatically generated prompts. In Proceedings of the 2020 Conference on Empirical Methods in Natural Language Processing, EMNLP 2020, Online, November 16-20, 2020, pages 4222-4235. Association for Computational Linguistics.

Eugen Slutzky. 1937. The summation of random causes as the source of cyclic processes. Econometrica: Journal of the Econometric Society, pages 105-146.

Ashish Vaswani, Noam Shazeer, Niki Parmar, Jakob Uszkoreit, Llion Jones, Aidan N. Gomez, Lukasz Kaiser, and Illia Polosukhin. 2017. Attention is all you need. In Advances in Neural Information Processing Systems 30: Annual Conference on Neural Information Processing Systems 2017, December 49, 2017, Long Beach, CA, USA, pages 5998-6008.
Herman Wold. 1938. A study in the analysis of stationary time series. Ph.D. thesis, Almqvist \& Wiksell.

Yonghui Wu, Mike Schuster, Zhifeng Chen, Quoc V. Le, Mohammad Norouzi, Wolfgang Macherey, Maxim Krikun, Yuan Cao, Qin Gao, Klaus Macherey, Jeff Klingner, Apurva Shah, Melvin Johnson, Xiaobing Liu, Lukasz Kaiser, Stephan Gouws, Yoshikiyo Kato, Taku Kudo, Hideto Kazawa, Keith Stevens, George Kurian, Nishant Patil, Wei Wang, Cliff Young, Jason Smith, Jason Riesa, Alex Rudnick, Oriol Vinyals, Greg Corrado, Macduff Hughes, and Jeffrey Dean. 2016. Google's neural machine translation system: Bridging the gap between human and machine translation. CoRR, abs/1609.08144.

Jingjing $\mathrm{Xu}$, Xuancheng Ren, Junyang Lin, and Xu Sun. 2018. Diversity-promoting GAN: A crossentropy based generative adversarial network for diversified text generation. In Proceedings of the 2018 Conference on Empirical Methods in Natural Language Processing, pages 3940-3949, Brussels, Belgium. Association for Computational Linguistics.

G Udny Yule. 1926. Why do we sometimes get nonsense-correlations between time-series?-a study in sampling and the nature of time-series. Journal of the royal statistical society, 89(1):1-63.

Han Zhang, Ian J. Goodfellow, Dimitris N. Metaxas, and Augustus Odena. 2019. Self-attention generative adversarial networks. In Proceedings of the 36th International Conference on Machine Learning, ICML 2019, 9-15 June 2019, Long Beach, California, USA, volume 97 of Proceedings of Machine Learning Research, pages 7354-7363. PMLR. 